Pacific Journal of Mathematic 


\title{
DIRICHLET MULTIPLICATION IN LATTICE POINT PROBLEMS. II
}

\author{
J. P. TULL
}

1. The author $[8]^{*}$ has given a theorem in which we assume two functions $A$ and $B$ have asymptotic formulae of the form

$$
A(x)=\sum_{\mu=1}^{h} x^{\alpha}{ }_{\mu} P_{\mu}(\log x)+O\left\{x^{\alpha} \log ^{l}(x+1)\right\}
$$

and upper estimates $O\left\{x^{\rho} \log ^{m}(x+1)\right\}$ on their total variations. We then conclude that their Stieltjes resultant $C$ satisfies a formula similar to (1). The $\alpha_{\mu}$ are complex numbers, the $P_{\mu}$ are polynomial functions, and we give an explicit formula for the error term in the resultant in terms of the given parameters.

In this paper we shall give a generalization of the above-mentioned result which will cover a wider class of lattice point problems.

2. Given two functions $A$ and $B$ defined for $x \geqq 1$, of bounded variation on each bounded interval, we call the Stieltjes resultant of $A$ by $B$ any function $C$ such that

$$
C(x)=\int_{1}^{x} A(x / u) d B(u)
$$

wherever the integral exists and for all $x$ either.

$$
\lim _{h \rightarrow 0+} C(x-h) \leqq C(x) \leqq \lim _{h \rightarrow 0+} C(x+h)
$$

or

$$
\lim _{h \rightarrow 0+} C(x+h) \leqq C(x) \leqq \lim _{h \rightarrow 0+} C(x-h) .
$$

Note that there are at most countably many $x$ for which the integral (1) does not exist, namely those $x=a b$ where $a$ is a discontinuity of $A$ and $b$ is a discontinuity of $B$. Note further that if $A(1)=B(1)=0$ then the Stieltjes resultant is a commutative binary operation:

$$
\int_{1}^{x} A(x / u) d B(u)=\int_{1}^{x} B(x / u) d A(u) \text {. }
$$

Widder [9] gives a slightly more restrictive definition of Stieltjes resultant, however, his requirement that $A, B$, and $C$ be "normalized" is unnecessary.

Received August 19, 1958. Excerpt from dissertation for the degree Doctor of Philosophy, University of Illinois, 1957.

* Note that in the proof of [8] Theorem 2, no use was made of the assumption $\alpha, \beta$, $\rho, \tau$ are non-negative. Thus that assumption may be deleted from the theorem. 
We shall denote the total variation of a function $A$ on $(1, x)$ by $V_{A}(x)$.

3. In place of the functions $P_{\mu}(\log x)$ in the asymptotic formulae we shall use the more general slowly oscillating functions. By "slowly oscillating function" we mean a function $L$ which is positive-valued and continuous on $x \geqq x_{0}$, for some $x_{0} \geqq 0$, such that

$$
\lim _{x \rightarrow \infty} L(c x) / L(x)=1
$$

for every $c>0$. Karamata [4] has characterized such functions in the form

$$
L(x)=a \rho(x) e^{\int_{x_{0}}^{x} t^{-1} \delta(t) d t}
$$

where $a>0, \rho(x) \rightarrow 1, \delta(x) \rightarrow 0$ as $x \rightarrow \infty ; \rho$ and $\delta$ being continuous on $x \geqq x_{0}$. (See also a proof by Korevaar et al [5]).

From (2) it follows that the limit in (1) is uniform in $c$ on each bounded interval

$$
0<k_{1} \leqq c \leqq k_{2} .
$$

Korevaar et al actually proved the uniformity first then used this property in proving the characterization (2).

Now a wide class of slowly oscillating functions can be written in the special form

$$
L(x)=a e^{\int_{1^{t}-1}^{x^{-1} \delta(t) d t} .} .
$$

We shall give such functions the name "special slowly oscillating function". However, we require in (4) only that $a>0$ and $\delta(x) \rightarrow 0$ as $x \rightarrow \infty$, where $\delta$ is bounded and Lebesgue integrable on each bounded interval. The function $\delta$ need not be continuous.

In order to maintain smoothness and to get a definite result we find it necessary-and quite reasonable-to deal mainly with special slowly oscillating functions.

For applications see Bateman [1], [2] and Bateman and Grosswald [3].

4. THeorem. Suppose $A$ and $B$ are complex valued function on $(1, \infty)$ of bounded variation on each bounded interval and with $A(1)=$ $B(1)=0 . \quad$ Suppose

$$
R(x)=\sum_{\mu=1}^{m} c_{\mu} x^{\alpha}{ }_{\mu} L_{\mu}(x)
$$

and

$$
S(x)=\sum_{\nu=1}^{n} d_{\nu} x^{\beta} M_{\nu}(x)
$$


with

$$
V_{R}(x)=O\left\{V_{A}(x)\right\}, V_{S}(x)=O\left\{V_{B}(x)\right\}
$$

where the $c_{\mu}, \alpha_{\mu}, d_{\nu}, \beta_{\nu}$ are complex numbers such that if $\mathfrak{R}\left(\alpha_{\mu}\right)=\mathfrak{R}\left(\beta_{\nu}\right)$ then $\alpha_{\mu}=\beta_{\nu}$, and the $L_{\mu}$ and $M_{\nu}$ are special slowly oscillating functions.

We assume $R$ and $S$ so chosen that there exist $\alpha, \beta$ with $\alpha \leqq \Re\left(\alpha_{\mu}\right)$ for each $\mu, \beta \leqq \Re\left(\beta_{\nu}\right)$ for each $\nu$, and there exist slowly oscillating functions $L_{0}, M_{0}$ such that

$$
\begin{aligned}
& \Delta_{1}(x)=A(x)-R(x)=O\left\{x^{\alpha} L_{0}(x)\right\}, \\
& \Delta_{2}(x)=B(x)-S(x)=O\left\{x^{\beta} M_{0}(x)\right\} .
\end{aligned}
$$

Further, we assume that there exist $\rho \geqq \alpha, \tau \geqq \beta$ and slowly oscillating functions $P_{0}$ and $Q_{0}$ such that

$$
V_{A}(x)=O\left\{x^{\rho} P_{0}(x)\right\}, V_{B}(x)=O\left\{x^{\tau} Q_{0}(x)\right\} .
$$

If $C$ is the Stieltjes resultant of $A$ and $B$ then there is a function $T$ of the form (1) such that if $\rho>\beta$ and $\tau>\alpha, 1 \leqq y \leqq x, z=x / y$,

(5) $\quad C(x)=T(x)+O\left\{x^{x} L_{0}(x)\right\}+O\left\{x^{\beta} M_{0}(x)\right\}$

$$
\begin{aligned}
& +O\left\{x^{\alpha} \sum_{\Re\left(\beta_{\nu}\right)=\alpha} \int_{1}^{x} u^{-1} M_{\nu}(u) L_{0}(x / u) d u\right\}+O\left\{x^{\beta} \sum_{\Re\left(x_{\mu}\right)=\beta} u^{-1} L_{\mu}(u) M_{0}(x / u) d u\right\} \\
& +O\left\{z^{\alpha} y^{\tau} L_{0}(z) Q_{0}(y)\right\}+O\left\{z^{p} y^{\beta} M_{0}(y) P_{0}(z)\right\}
\end{aligned}
$$

the O-constants being independent of $x, y$ and $z$. More specifically,

$$
\begin{aligned}
T(x)= & \int_{1}^{x} R(x / u) d S(u)+\sum_{\Re\left(\beta_{\nu}\right)>\infty} d_{\nu} x^{\beta_{\nu}} N_{\nu}(x) \\
& +\sum_{\Re\left(\alpha_{\mu}\right)>\beta} c_{\mu} x^{\alpha_{\mu}} N_{\mu}^{\prime}(x)-\Delta_{2}(1) \sum_{\Re\left(\alpha_{\mu}\right)>\beta} c_{\mu} x^{x_{\mu}} L_{\mu}(x)
\end{aligned}
$$

where

$$
\int_{1}^{x} R(x / u) d S(u)=\sum_{\mu, \nu} c_{\mu} d_{\nu} \int_{1}^{x}(x / u)^{x}{ }_{\mu} L_{\mu}(x / u) d\left(u^{\beta} \nu M_{\nu}(u)\right)
$$

is a complex linear combination of functions

$$
x^{\gamma} \mu_{\nu} L_{\mu \nu}(x)
$$

with $\gamma_{\mu \nu}=\alpha_{\mu}$ or $\beta_{\nu}$, whichever has the greater real part, $L_{\mu \nu}$ a slowly oscillating function; and

$$
\begin{aligned}
& N_{\nu}(x)=x^{-\beta_{\nu}} \int_{1}^{x} \Delta_{1}(x / u) d\left(u^{\beta_{\nu}} M_{\odot}(u)\right) \\
& N_{\mu}^{\prime}(x)=x^{-\alpha_{\mu}} \int_{1}^{x} \Delta_{2}(x / u) d\left(u^{\alpha_{\mu}} L_{\mu}(u)\right)
\end{aligned}
$$


are linear combinations of slowly oscillating functions. Further, the functions $L_{\mu \nu}, N_{\mu}^{\prime}, N_{\nu}$ are absolutely continuous on each bounded interval $(1, x)$.

If $\tau=\alpha$ the error is then

$$
\begin{aligned}
& O\left\{x^{\beta} M_{0}(x)\right\}+O\left\{x^{\tau} \int_{1}^{x} u^{-1} Q_{0}(u) L_{0}(x / u) d u\right\} \\
& +O\left\{x^{\beta} \sum_{\Re\left(x_{\mu}\right)=\beta} \int_{1}^{x} u^{-1} L_{\mu}(u) M_{0}(x / u) d u\right\}
\end{aligned}
$$

the integrals in the error terms being absolutely continuous slowly ocsillating functions.

If $\alpha>\tau$ we have simply

$$
C(x)=T(x)+O\left\{x^{\alpha} L_{0}(x)\right\}
$$

$T(x)$ being, in these latter two cases, the same as in (6) excepting that the term

$$
\sum_{\Re\left(\beta_{\nu}\right)>\alpha} d \nu x^{\circ} \nu N,(x)
$$

is absorbed in the error term. The cases $\rho \leqq \beta$ are similar.

Finally,

$$
V_{C}(x)=O\left\{x^{\max (\rho, \tau)} S_{0}(x)\right\}
$$

with

$$
S_{0}(x)= \begin{cases}P_{0}(x), & \text { if } \rho>\tau, \\ Q_{0}(x), & \text { if } \rho<\tau, \\ \int_{1}^{x} u^{-1} P_{0}(u) Q_{0}(x / u) d u, & \text { if } \rho=\tau .\end{cases}
$$

Note that if there are different $\alpha_{\mu}, \beta$, with equal real parts, then the theorem still holds with the exception that the resulting integral

$$
\int_{1}^{x}(x / u)^{\alpha}{ }_{\mu} L_{\mu}(x / u) d\left(u^{\beta_{\nu}} M_{\nu}(u)\right)
$$

in $T(x)$ is not necessarily a combination of powers of $x$ times slowly oscillating functions.

5. It suffices to consider only those values of $x>1$ for which the integral defining the Stieltjes resultant converges. For any such $x$,

$$
C(x)=\int_{1}^{x} A(x / u) d B(u)=I_{1}+I_{2}+I_{3}+I_{4},
$$

where 


$$
\begin{aligned}
& I_{1}=\int_{1}^{x} R(x / u) d S(u), \\
& I_{2}=\int_{1}^{x} \Delta_{1}(x / u) d S(u), \\
& I_{3}=\int_{1}^{x} R(x / u) d \Delta_{2}(u), \\
& I_{4}=\int_{1}^{x} \Delta_{1}(x / u) d \Delta_{2}(u) .
\end{aligned}
$$

The proof now consists of demonstrating several lemmas which amount to the fact that if $I_{1}$ and $I_{2}$ are split up suitably, as indicated in formula (4.6), then the right hand side of (4.6) is a sum of functions $a x^{\gamma} N(x)$ where $N$ is an absolutely continuous slowly oscillating function, and the remaining terms are of the order of magnitude stated in (4.5), (4.7), or (4.8) as the case may be. We shall give here the two lemmas used to demonstrate the estimates on the error terms given in $(4.5,7$, $8,9)$. The remainder of the proof then involves juggling terms by means of integration by parts and considering various cases according to whether

$$
\Re\left(\beta_{\nu}\right)>,=\text {, or }>\alpha \text { and } \Re\left(\alpha_{\mu}\right)>,=,<\beta .
$$

Lemma 1. If $L$ and $M$ are slowly oscillating functions (on $x \geqq 1$ ) and $\gamma>0$, then for $1 \leqq y \leqq x, q \geqq 1$,

$$
\int_{q}^{y} u^{\gamma-1} M(u) L(x / u) d u=O\left\{y^{\gamma} M(y) L(x / y)\right\},
$$

the O-constant depending only on $L, M$, and $\gamma$.

Since $L$ and $M$ are positive functions we may assume $q=1$. Now if

$$
\begin{aligned}
& L(x)=a \rho(x) \exp \int_{1}^{x} t^{-1} \delta(t) d t \\
& M(x)=b \tau(x) \exp \int_{1}^{x} t^{-1} \varepsilon(t) d t
\end{aligned}
$$

where $\rho(x) \rightarrow 1, \tau(x) \rightarrow 1, \delta(x) \rightarrow 0, \varepsilon(x) \rightarrow 0$, as $x \rightarrow \infty, a>0, b>0$, then let

$$
J(x)=L(x) / \rho(x), \quad K(x)=M(x) / \tau(x)
$$

so that

$$
L(x) \sim J(x), \quad M(x) \sim K(x)
$$

as $x \rightarrow \infty$.

Without loss of generality, we may assume that for all $t \geqq 1$,

$$
|\delta(t)| \leqq \gamma / 4, \quad|\varepsilon(t)| \leqq \gamma / 4
$$


for otherwise, the excess, which occurs only on a bounded interval, can be obsorbed into the coefficient functions $a \rho(x)$ and $b \tau(x)$.

Now clearly

$$
\int_{1}^{y} u^{\gamma-1} M(u) L(x / u) d u=O\left\{\int_{1}^{y} u^{\gamma-1} K(u) J(x / u) d u\right\} .
$$

Upon integrating by parts we have

$$
\begin{aligned}
& \int_{1}^{y} u^{\gamma-1} K(u) J(x / u) d u \\
&= \frac{1}{\gamma}\left\{y^{\gamma} K(y) J(x / y)-K(1) J(x)\right\} \\
& \quad-\frac{1}{\gamma} \int_{1}^{y} u^{\gamma-1} K(u) \varepsilon(u) J(x / u) d u \\
&+\frac{1}{\gamma} \int_{1}^{y} u^{\gamma-1} K(u) J(x / u) \delta(x / u) d u \\
& \leqq \frac{1}{\gamma}\left\{y^{\gamma} K(y) J(x / y)-K(1) J(x)\right\} \\
&+\frac{1}{2} \int_{1}^{y} u^{\gamma-1} K(u) J(x / u) d u
\end{aligned}
$$

by (3). Thus

$$
\begin{aligned}
\int_{1}^{y} u^{\gamma-1} K(u) J(x / u) d u & \leqq \frac{2}{\gamma}\left\{y^{\gamma} K(y) J(x / y)-K(1) J(x)\right\} \\
\leqq \frac{2}{\gamma} y^{\gamma} K(y) J(x / y) & =O\left\{y^{\gamma} M(y) L(x / y)\right\} .
\end{aligned}
$$

This completes the proof.

With the aid of lemma 1 and some simple inequalities one can readily prove the following.

Lemma 2. If $A$ and $B$ are functions of bounded variation on $(1, x)$ for each $x>1$ and if for $x \geqq 1$,

$$
A(x)=O\left(x^{x} L(x)\right), \quad V_{B}(x)=O\left(x^{\tau} Q(x)\right),
$$

where $L$ and $Q$ are slowly oscillating functions on $x \geqq 1$ and $\alpha$ and $\tau$ are real numbers, then for $2 \leqq y \leqq x, z=x / y$, if $x$ is not a product of a discontinuity of $A$ by a discontinuity of $B$

$$
\int_{1}^{y} A(x / u) d B(u)= \begin{cases}O\left\{z^{\alpha} y^{\tau} L(z) Q(y)\right\} & (\tau>\alpha) \\ O\left\{x^{\alpha} \int_{1}^{y} u^{-1} Q(u) L(x / u) d u\right\} & (\tau=\alpha) \\ O\left\{z^{\alpha} y^{\tau} L(z) Q(y)\right\}+O\left\{x^{\alpha} L(x)\right\} & (\tau<\alpha)\end{cases}
$$


uniformly in $x$ and $y$

6. It is fairly easy to show that Theorem 2 of [8] is a corollary to the theorem of the present paper.

As was mentioned in paper [8] the author has given an example [7] which shows that Landau's theorem [6] on the multiplication of Dirichlet series gives the best possible result. Further, we showed that the same example applies to the result of [8] so that the exponent of $x$ in the error term is as a general theorem best possible if we restrict the main terms to the form in (1.1). This gives some indication of the strength of our result, however, we are as yet unable to decide whether the result with the more general main terms of the present paper is improvable.

The author wishes to give due credit to his teacher, P. T. Bateman, who gave valuable help in getting this paper started.

\section{REFERENCES}

1. P. T. Bateman, Remark on problem 4459, Amer. Math. Monthly, 61 (1954), 477-479.

2. -_- - Proof of a conjecture of Grosswald, Duke Math. J. 25, No. 1 (1958), 67-72.

3. - and E. Grosswald, On a theorem of Erdös and Szekeres, Illinois J. Math. 2 (1958), 88-98.

4. J. Karamata, Sur un mode de croissance reguliere des fonctions, Math. (Cluj) 4 (1930), $38-53$.

5. J., Korevaar, T. van Aardenne-Ehrenfest, and N. G. de Bruijn, A note on slowly oscillating functions, Nieuw Arch. Wiskunde 23 (1949), 77-86.

6. E., Landau, Handbuch der Lehre von der Verteilung der Primzahlen, Leipzig 11 (1909), 755-758.

7. J. P. Tull, The mutiplication problem for Dirichlet series, Proc. Amer. Math. Soc.

9, No. 2 (1958), 332-334.

8. , Dirichlet multiplication in latice point problems, Duke Math. J. 26, No.

1, (1959), 73-80.

9. D. V. Widder, The Laplace Transform, Princeton (1941), 83-91.

The Ohio State University 



\section{PACIFIC JOURNAL OF MATHEMATICS}

\section{EDITORS}

\section{David Gilbarg}

Stanford University

Stanford, California

\section{R. A. Beaumont}

University of Washington

Seattle 5 , Washington

\author{
A. L. Whiteman
}

University of Southern California Los Angeles 7, California

L. J. Paige

University of California

Los Angeles 24, California

\author{
E. F. BECKENBACH \\ C. E. BURGESS \\ E. HEWITT \\ A. HORN
}

\author{
V. GANAPATHY IYER \\ R. D. JAMES \\ M. S. KNEBELMAN \\ L. NACHBIN
}

ASSOCIATE EDITORS
I. NIVEN

T. G. OSTROM

H. L. ROYDEN

M. M. SCHIFFER
E. G. STRAUS

G. SZEKERES

F. WOLF

K. YOSIDA

\section{SUPPORTING INSTITUTIONS}

\author{
UNIVERSITY OF BRITISH COLUMBIA \\ CALIFORNIA INSTITUTE OF TECHNOLOGY \\ UNIVERSITY OF CALIFORNIA \\ MONTANA STATE UNIVERSITY \\ UNIVERSITY OF NEVADA \\ OREGON STATE COLLEGE \\ UNIVERSITY OF OREGON \\ OSAKA UNIVERSITY \\ UNIVERSITY OF SOUTHERN CALIFORNIA
}

\author{
STANFORD UNIVERSITY \\ UNIVERSITY OF TOKYO \\ UNIVERSITY OF UTAH \\ WASHINGTON STATE COLLEGE \\ UNIVERSITY OF WASHINGTON \\ * * * \\ AMERICAN MATHEMATICAL SOCIETY \\ CALIFORNIA RESEARCH CORPORATION \\ HUGHES AIRCRAFT COMPANY \\ SPACE TECHNOLOGY LABORATORIES
}

Mathematical papers intended for publication in the Pacific Journal of Mathematics should be typewritten (double spaced), and the author should keep a complete copy. Manuscripts may be sent to any one of the four editors. All other communications to the editors should be addressed to the managing editor, L. J. Paige at the University of California, Los Angeles 24, California.

50 reprints per author of each article are furnished free of charge; additional copies may be obtained at cost in multiples of 50 .

The Pacific Journal of Mathematics is published quarterly, in March, June, September, and December. The price per volume (4 numbers) is $\$ 12.00$; single issues, $\$ 3.50$. Back numbers are available. Special price to individual faculty members of supporting institutions and to individual members of the American Mathematical Society: $\$ 4.00$ per volume; single issues, $\$ 1.25$.

Subscriptions, orders for back numbers, and changes of address should be sent to Pacific Journal of Mathematics, 2120 Oxford Street, Berkeley 4, California.

Printed at Kokusai Bunken Insatsusha (International Academic Printing Co., Ltd.), No. 6, 2-chome, Fujimi-cho, Chiyoda-ku, Tokyo, Japan.

PUBLISHED BY PACIFIC JOURNAL OF MATHEMATICS, A NON-PROFIT CORPORATION

The Supporting Institutions listed above contribute to the cost of publication of this Journal, but they are not owners or publishers and have no responsibility for its content or policies. 


\section{Pacific Journal of Mathematics}

\section{Vol. 9, No. $2 \quad$ June, 1959}

Lee William Anderson, On the breadth and co-dimension of a topological lattice

Frank W. Anderson and Robert L. Blair, Characterizations of certain lattices

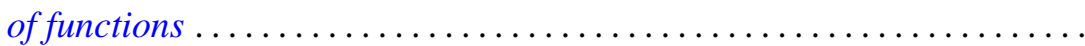

Donald Charles Benson, Extensions of a theorem of Loewner on integral

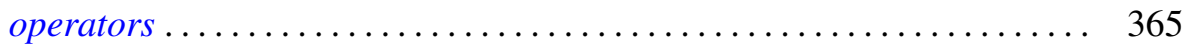

Errett Albert Bishop, A duality theorem for an arbitrary operator ........ 379

Robert McCallum Blumenthal and Ronald Kay Getoor, The asymptotic distribution of the eigenvalues for a class of Markov operators ........

Delmar L. Boyer and Elbert A. Walker, Almost locally pure Abelian

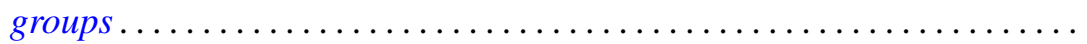

Paul Civin and Bertram Yood, Involutions on Banach algebras ........... Lincoln Kearney Durst, Exceptional real Lehmer sequences .... 415

Eldon Dyer and Allen Lowell Shields, Connectivity of topological

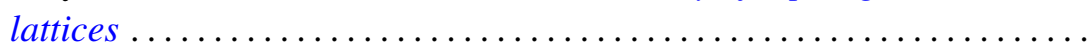

Ronald Kay Getoor, Markov operators and their associated

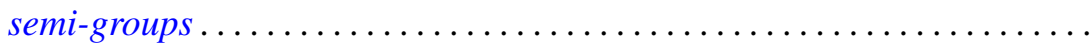

Bernard Greenspan, A bound for the orders of the components of a system of algebraic difference equations

Branko Grünbaum, On some covering and intersection properties in

Minkowski spaces ............................

Bruno Harris, Derivations of Jordan algebras ..............

Henry Berge Helson, Conjugate series in several variables.

Isidore Isaac Hirschman, Jr., A maximal problem in harmonic analysis.

II .

Alfred Horn and Robert Steinberg, Eigenvalues of the unitary part of a matrix

Edith Hirsch Luchins, On strictly semi-simple Banach algebras ...

William D. Munro, Some iterative methods for determining zeros of

functions of a complex variable...

John Rainwater, Spaces whose finest uniformity is metric .

William T. Reid, Variational aspects of generalized convex functions ....

A. Sade, Isomorphisme d'hypergroupoï des isotopes ...... . .

Isadore Manual Singer, The geometric interpretation of a special

connection . . .

Charles Andrew Swanson, Asymptotic perturbation series for characteristic

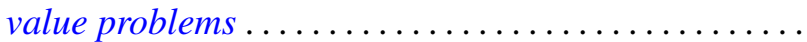

